\title{
A atuação da laserterapia na angiogênese e no reparo tecidual
}

\author{
The performance of phototherapy in angiogenesis and tissue repair \\ El rendimiento de laserterapia en angiogénesis y reparación de tejidos
}

Recebido: 26/02/2021 | Revisado: 07/03/2021 | Aceito: 11/03/2021 | Publicado: 18/03/2021

\author{
Alieny Cristina Duarte Ferreira \\ ORCID: https://orcid.org/0000-0003-0370-3177 \\ Universidade Estadual da Paraíba, Brasil \\ E-mail: alienycris@hotmail.com \\ Ana Luzia Araújo Batista \\ ORCID: https://orcid.org/0000-0002-7780-060X \\ Universidade Estadual da Paraíba, Brasil \\ E-mail: analuziabatista@globo.com \\ Maria Helena Chaves de Vasconcelos Catão \\ ORCID: https://orcid.org/0000-0001-7681-3225 \\ Universidade Estadual da Paraíba, Brasil \\ E-mail: mhelenact@zipmail.com.br
}

\begin{abstract}
Resumo
A cicatrização de feridas é um processo complexo, composto por vários estágios interdependentes e simultâneos com o objetivo de reparar o tecido e, dentre os métodos que utilizados, a laserterapia apresenta efeitos estimuladores biológicos e regenerativos. O objetivo dessa revisão é compreender o mecanismo de ação da laserterapia na reparação tecidual e, mais especificamente, na angiogênese. Foi realizada uma pesquisa bibliográfica nas bases de dados PUBMED, LILACS e SCIELO sobre o assunto, utilizando as seguintes palavras-chave: "fototerapia e angiogênese" e "fototerapia e cicatrização de feridas". Nessa busca, foram totalizados 3634 artigos que após análise dos critérios, foram excluídos 3624, restando 10 trabalhos. Diante do exposto, pode-se concluir que a laserterapia tem se apresentado como uma alternativa terapêutica na reparação tecidual, atuando como fotobiomodulador, com efeitos analgésicos, anti-inflamatórios, anti-edema e antimicrobianos, oferecendo melhorias no mecanismo de resposta celular e apresentando vantagens nas três etapas do processo de cicatrização.
\end{abstract}

Palavras-chave: Fototerapia; Neovascularização fisiológica; Cicatrização de feridas.

\begin{abstract}
Wound healing is a complex process, composed of several interdependent and simultaneous stages to repair the tissue and, among the methods used, laser therapy has biological and regenerative stimulatory effects. The aim of this review is to understand the mechanism of action of laser therapy in tissue repair and, more specifically, angiogenesis. A bibliographic search was performed in the databases PUBMED, LILACS and SCIELO on the subject, using the following keywords: "phototherapy and angiogenesis" and "phototherapy and wound healing". In this search, 3634 articles were totaled, which after analysis of the criteria, 3624 were excluded, remaining 10 papers. In view of the above, it can be concluded that laser therapy has been presented as a therapeutic alternative in tissue repair, acting as a photobiomodulator, with analgesic, anti-inflammatory, anti-edema and antimicrobial effects, offering improvements in cellular response mechanisms and presenting advantages in the three stages of the healing process.
\end{abstract}

Keywords: Phototherapy; Neovascularization, physiologic; Wound healing.

\section{Resumen}

La cicatrización de heridas es un proceso complejo, compuesto por varias etapas interdependientes y simultáneas para reparar el tejido y, entre los métodos utilizados, la terapia con láser tiene efectos estimulantes biológicos y regenerativos. El propósito de esta revisión es comprender el mecanismo de acción de la terapia con láser en la reparación de tejidos y, más específicamente, en la angiogénesis. Se realizó una búsqueda bibliográfica en las bases de datos PUBMED, LILACS y SCIELO sobre el tema, utilizando las siguientes palabras clave: "fototerapia y angiogénesis" y "fototerapia y cicatrización de heridas". En esta búsqueda se agregaron un total de 3634 artículos, los cuales, luego de analizar los criterios, se excluyeron 3624, quedando 10 estudios. Dado lo anterior, se puede concluir que la terapia con láser se ha presentado como una alternativa terapéutica en la reparación de tejidos, actuando como fotobiomodulador, con efectos analgésicos, antiinflamatorios, antiedema y antimicrobianos, ofreciendo mejoras en los mecanismos de respuesta celular y presenta ventajas en las tres etapas del proceso de curación.

Palabras clave: Fototerapia; Neovascularización fisiológica; Cicatrización de la herida. 


\section{Introdução}

A cicatrização de feridas é um processo complexo que envolve a organização de células, sinais químicos e matriz extracelular. É composta de uma série de estágios complexos, interdependentes e simultâneos com o objetivo de reparar o tecido e o seu tratamento tem como objetivo primordial o fechamento rápido da lesão de forma a se obter cicatriz funcional e esteticamente satisfatória (Mendonça \& Coutinho-Netto, 2009).

Atualmente, existem métodos para aliviar o processo inflamatório, proporcionando melhoria na nutrição celular e favorecendo o reparo tecidual danificado (Melo et al., 2011). O uso de terapias alternativas não medicinais para corrigir os processos de regeneração reparadora em várias condições patológicas é um dos problemas mais urgentes da Medicina Moderna (Iryanov, 2016).

A Laserterapia tem sido utilizada na última década para tratar uma variedade de lesões teciduais, especialmente as feridas infectadas, inflamadas e hipóxicas. A terapia com laser de diferentes tipos é amplamente utilizada na prática clínica e visa proporcionar os efeitos anestésicos, anti-edematosos, antiinflamatórios e tróficos-estimulantes. Além do mais, é um tratamento confortável, não-invasivo, asséptico, indolor, inofensivo e controlável (Iryanov, 2016), sendo bem tolerada por tecidos biológicos e sem associação com efeitos Deletérios (Fiório et al., 2011).

O laser de baixa intensidade (LBI) restaura a capacidade funcional do tecido, com base em efeitos estimulativos biológicos e regenerativos (Sawasaki et al., 2009), sendo apresentado como uma alternativa para o tratamento da ferida devido aos seus mecanismos (Corazza et al., 2007; Dall et al., 2009). Portanto, considerando a relevância de compreender melhor o processo biológico de atuação da laserterapia na angiogênese realizou-se uma revisão de literatura dando enfoque ao processo da ferida, reparo tecidual e mecanismo celular.

\section{Metodologia}

Foi realizada uma busca na literatura de acordo com a metodologia de Pereira et al. (2018), nos bancos de dados PUBMED, LILACS e SCIELO acerca do tema abordado e com método qualitativo. Selecionamos estas bases de dados pelo fato de serem completas em revistas científicas que passam por avaliação periódica realizada por comitê composto por profissionais experientes, representantes das grandes áreas de conhecimento.

Foram utilizadas as seguintes palavras-chave: "phototherapy and angiogenesis" e " phototherapy and wound healing", foram encontrados 3.634 artigos. Após a leitura dos resumos, foram excluídos os temas divergentes ao foco da análise, os artigos anteriores a 2007, teses e dissertações e artigos em português. Portanto, ao final foram totalizados 30 artigos, porém 20 deles estavam em duplicata, finalizando 10 artigos. $\mathrm{Na}$ exposição do tema em questão, preferiu-se topificar da seguinte forma: a ferida e o reparo tecidual, angiogênese, mecanismo celular e laserterapia e o processo cicatricial.

\section{Revisão de Literatura}

\section{A ferida e o reparo tecidual}

A ferida é uma lesão física corporal caracterizada pela ruptura da continuidade normal das estruturas do corpo (Hussein et al., 2011) que representa a interrupção, anatômica ou funcional, da continuidade de um determinado tecido que é seguido de dano ou morte celular (Pinheiro et al., 2009). A descontinuidade da integridade do tecido pode ser induzida por trauma físico, químico ou mecânico (Sousa et al., 2015) ou desencadeada por uma condição médica, a exemplo dos procedimentos cirúrgicos (Melo et al., 2011; Chaves et al., 2014).

A cicatrização das feridas é um processo complexo que requer a coordenação de vários eventos, incluindo coagulação, inflamação, formação de tecido de granulação, epitelização, angiogênese, síntese de colágeno e remodelação de tecidos (Fiório 
et al., 2011; Lim et al., 2011). Neste processo, ocorre uma interação complexa entre células, matriz extracelular, vasos sanguíneos, proteases, citocinas e quimiocinas (Gonçalves et al., 2010). O reparo de tecidos é um processo interativo, envolvendo mediadores químicos, células e a resposta inflamatória, caracterizada pelas etapas clássicas de reparo: inflamação, granulação e remodelação (Colombo et al., 2013).

As falhas de reparo mais importantes são aquelas que ocorrem nos estágios iniciais o que leva à acentuação do edema, redução da proliferação vascular e diminuição das quantidades de elementos celulares, como leucócitos, macrófagos e fibroblastos (Carvalho et al., 2010). Os fatores que afetam o reparo tecidual, ou seja, que influenciam a resposta inflamatória pode ser dividido em locais e sistêmicos incluindo: deficiências nutricionais, enzimas ativas, antioxidantes, vitaminas, minerais e aminoácidos, por alterarem a regeneração do tecido através de interferência com reações inflamatórias e função imunológica (Pinheiro et al., 2009). Condições físicas e alterações sistêmicas como diabetes mellitus, obesidade, desnutrição, idade avançada (mais de 60 anos), diminuição da perfusão, malignidade, falência de órgãos, sepse e até restrições de mobilidade têm impacto na cicatrização (Chaves et al., 2015).

$\mathrm{Na}$ cicatrização de feridas, a proliferação celular começa caracteristicamente das margens da ferida e se estende ao seu centro, principalmente por causa da baixa disponibilidade de nutrientes e oxigênio neste site (Ribeiro, et al., 2015). Portanto, o tamanho e a profundidade da ferida é um fator importante a se considerar na análise do reparo e na comparação entre estudos.

O processo fisiológico do reparo tecidual envolve múltiplos fatores, sendo a manutenção de suas fases reforçadas por agentes exógenos que variam de biomoléculas - fatores de crescimento, extratos naturais, a modalidades físicas - campos elétricos, oxigênio hiperbárico (Arany et al., 2007).

De acordo com as fases da cicatrização, na primeira delas, a fase inflamatória, há extravasamento sanguíneo, com a presença de plasma e elementos celulares, principalmente plaquetas, ocupando o local da injúria (Moura et al., 2014). A agregação plaquetária e a coagulação sanguínea geram um tampão rico em fibrina, que além de restabelecer a hemostasia e formar uma barreira contra a invasão de microrganismos, organiza a matriz provisória necessária para a migração celular e que também servirá como reservatório de citocinas e fatores de crescimento que serão liberados durante as fases seguintes do processo cicatricial (Mendonça \& Coutinho-Netto, 2009). Ainda nessa fase, ocorre uma modulação celular com uma diminuição da inflamação celular e um aumento na liberação de fatores de crescimento, dando início a póxima fase, a proliferativa (Corazza et al., 2007).

A fase proliferativa consiste na reepitelização, que se inicia horas após a lesão, com a movimentação das células epiteliais oriundas tanto da margem como de apêndices epidérmicos localizados no centro da lesão, fibroplasia e angiogênese, compondo o chamado tecido de granulação (Mendonça \& Coutinho-Netto, 2009; Moura et al., 2014) e acontece entre o $5^{\circ} \mathrm{e} 14^{\circ}$ dia após a lesão. Nessa etapa, a ferida entra num período de maturação, durante o qual ocorre a remodelação, principalmente pela deposição de uma rede de fibra de colágeno mais madura e melhor organizada (Colombo et al., 2013). Os fibroblastos são estimulados a proliferar e migrar para o local da lesão, sintetizando colágeno e proteoglicanos com a finalidade de restaur a integridade do tecido (Carvalho et al., 2010) e, dessa forma, produzir a nova matriz extracelular necessária ao crescimento celular, enquanto os novos vasos sanguíneos carreiam oxigênio e nutrientes necessários ao metabolismo celular local (Mendonça \& Coutinho-Netto, 2009).

$\mathrm{Na}$ última fase, a de remodelagem, há uma tentativa de retorno da estrutura tecidual normal, com desenvolvimento dos elementos da matriz, levando ao depósito de proteoglicanos e colágeno (Moura et al., 2014). Dessa forma, a remodelação do tecido ocorre onde pela indução da neovascularização e intensa deposição e maturação de colágeno Portanto, a ferida é nutrida no início do processo de reparo pelos vasos remanescentes, localizados na periferia da lesão e para seu reparo completo o crescimento vascular é imprescindível (Corazza et al., 2007). 


\section{Angiogênese}

A angiogênese é um dos principais fatores relacionados ao reparo tecidual e está compreendida na segunda fase de cicatrização que corresponde uma etapa fundamental, a qual novos vasos sanguíneos são formados a partir de vasos preexistentes, ajudando a compor o tecido de granulação provisório (Mendonça \& Coutinho-Netto, 2009; Lim et al., 2011). A angiogênese restaura o nível de oxigênio e nutrientes para o tecido recém-formado, fornecendo a alta demanda metabólica, favorecendo a proliferação celular e migração, bem como a síntese protéica (Colombo et al., 2013).

A angiogênese está envolvida desde o início do processo de cicatrização e esse suprimento vascular permite um aumento na taxa metabólica e na atividade de mitose (Corazza et al., 2007) caracterizando o tecido de granulação envolvido no processo de cicatrização da pele (Fiório, et al., 2011; Dall et al., 2009).

A fase inicial da angiogênese está relacionada com a degradação da matriz extracelular para permitir a migração e recrutamento de células endoteliais. As enzimas essenciais para esse processo são produzidas e liberadas pelos mastócitos, incluindo triptase, elastase, catepsina $\mathrm{G}$ e colagenase (Pereira et al., 2010).

A angiogênese é estimulada no início do processo e mediada pela expressão induzida por hipóxia e a liberação de mediadores parácrinos. Isso explica o fato das EROs (espécies reativas de oxigênio), aumentada em condições de hipóxicas/isquêmicas funcionarem como importantes mediadores. Entretanto, em níveis elevados, podem atacar ácidos nucléicos, proteínas e fosfolípidos de membrana e, conseqüentemente, induzir a morte celular, apoptose e senescência (Lim et al., 2011). Além disso, elevados níveis de ácido lático e aminas bioativas também podem estimular a angiogênese (Mendonça \& Coutinho-Netto, 2009).

A indução da angiogênese foi inicialmente atribuída ao FGF (fator de crescimento fibroblástico) ácido ou básico. Subsequentemente, muitas outras moléculas foram identificadas como pró-angiogênicas, incluindo o VEGF (o fator de crescimento vascular endotelial), TGF- $\beta$ (fator de crescimento transformante beta), angiogenina, angiotropina e angiopoetina (Mendonça \& Coutinho-Netto, 2009).

Fatores de crescimento, proteínas, componentes das vias de coagulação/fibrinolítica, proteínas da matriz extracelular e plaquetas interagem com células endoteliais e pericitas adjacentes aos vasos sanguíneos, regulando a formação de novos vasos sanguíneos. Vários fatores de crescimento mostram potencial angiogênico, dentre eles o TGF- $\beta$ que é produzido pela maioria das células encontradas no tecido de granulação e aumenta a produção de outras citocinas, estimula a angiogênese e a produção de colágeno tipo I e inibe a produção de colagenase instersticial. Enquanto que, o VEGF desempenha um papel central na angiogênese, aumentando a permeabilidade vascular e a proliferação de células endoteliais vasculares (Lim et al., 2011).

\section{Mecanismo celular}

O reparo de tecido é um processo interativo, envolvendo mediadores químicos, células e respostas inflamatórias, caracterizada por passos clássicos de reparo: inflamação, granulação e remodelação. Todo o processo é desencadeado por uma injúria tecidual seguida imediatamente pela migração de neutrófilos para o local lesionado e consequentemente dos monócitos (Colombo, et al., 2013).

O processo de reparo tecidual é complexo e compreende alterações vasculares e celulares, proliferação epitelial e de fibroblastos, síntese e deposição de colágeno, produção de elastina e proteoglicanos, revascularização e contração da ferida. Destacam-se ainda, os efeitos trófico-regenerativos, anti-inflamatórios e analgésicos (Andrade, Clark \& Ferreira, 2014).

Os macrófagos são fundamentais para o processo de cicatrização de feridas e são responsáveis pela amplificação, orquestração e manutenção da resposta de cicatrização de feridas. Além de destruir bactérias e debridar tecido desvitalizado, eles secretam continuamente uma variedade de citocinas durante a fase inflamatória da cicatrização (Carvalho et al., 2010). 
Os mastócitos têm um papel fundamental no processo inflamatório e no reparo tecidual por produzirem e liberarem uma vasta gama de mediadores químicos que estão relacionados com a proliferação vascular e vasodilatação e também por otimizar o processo inflamatório. As células endoteliais e mastócitos produzem enzimas proteolíticas responsáveis pela degradação da membrana basal e da matriz extracelular, o que contribui para migração celular e formação de novos vasos (Pereira et al., 2010).

Os mastócitos podem participar ativamente no recrutamento de células polimorfonucleares através da produção de várias citocinas contidas nos grânulos citoplasmáticos e, dessa forma, contribuir para a amplificação e progressão do processo inflamatório, em parte por aumentar a concentração local de citocinas (Pereira et al., 2010).

A cicatrização da ferida começa com inflamação aguda, portanto neste processo a vasodilatação é seguida pelo aumento da permeabilidade vascular que tem a participação da histamina, um mediador inflamatório liberado por mastócitos presentes no tecido mucoso e conjuntivo. Assim, a degranulação dos mastócitos é um passo importante na cicatrização de feridas (Sawasaki et al., 2007).

Durante o processo de cicatrização, citocinas e fatores de crescimento são envolvidos na regulação da viabilidade celular e na remodelação da matriz extracelular. Inicialmente, numerosos produtos químicos são liberados para a área danificada causando alterações nos tecidos, consistentes com um processo inflamatório agudo (Melo et al., 2011).

O processo de cicatrização envolve migração celular inflamatória, síntese de tecido de granulação, deposição de colágeno e proteoglicano e maturação de cicatrizes, sendo associada à remodelação intensa (Gonçalves et al., 2010).

A reação inflamatória representa o primeiro evento a ocorrer após a lesão tecidual, cuja principal função é eliminar eventuais microorganismos e fornecer limpeza de feridas. Esta inflamação é absolutamente necessária para proporcionar cicatrização de feridas, embora sua persistência em longo prazo seja considerada uma das razões mais importantes para atrasar o processo de cicatrização (Melo et al., 2011).

A formação vascular pode ser associada à redução de células inflamatórias e à estimulação de fatores de crescimento angiogênicos através de macrófagos, linfócitos T, plaquetas, células endoteliais, fibroblastos e queratinócitos (Fiório et al., 2011; Corazza et al., 2007). Por esse motivo, nos estágios iniciais, os eventos biológicos, tais como a formação de novos vasos sanguíneos, associados à deposição progressiva e remodelação de fibras de colágeno, culminarão em um completo reparo cicatricial da área lesada (Melo et al., 2011).

O principal componente de uma cicatriz madura é o colágeno tipo I, e nas feridas cicatrizantes, os fibroblastos são recrutados e induzido a sintetizar colágeno em um processo chamado fibroplasia, iniciado pelo tecido de granulação na ferida e rica em colágeno tipo III. À medida que o processo de recuperação do tecido avança, o tecido de granulação é substituído por um tecido menos vascularizado e mais resistente rico em colágeno tipo I (Gonçalves et al., 2013).

Em condições patológicas, ocorre um aumento na concentração de óxido nítrico, o que facilita a formação de radicais livres, induzindo uma diminuição da respiração celular, bem como uma produção aumentada de EROs (Corazza et al., 2007). A situação de hipóxia e a liberação de mediadores parácrinos podem funcionar como mediadores importantes. Entretanto, EROs em níveis elevados, podem atacar ácidos nucléicos, proteínas e fosfolípidos de membrana e, conseqüentemente, induzir a morte celular, apoptose e senescência (Lim et al., 2011).

\section{Laserterapia e o Processo Cicatricial}

A fototerapia baseia-se no mecanismo da absorção da luz por um fotoreceptor para exercer efeitos sobre os sistemas biológicos vivos. A terapia com luz solar e a ultravioleta (UV) foi usada por muitas décadas, porém elas danificavam os tecidos quando da exposição prolongada. Nesse contexto, outras fontes de luz artificiais, incluindo luzes halógenas, foram inseridas, mas também causavam danos térmicos aos tecidos irradiados (Fushimi et al.,2012). 
Recentemente, a laserterapia tem se mostrado úteis para várias aplicações médicas, no entanto, os sistemas a laser são caros e requer reparos freqüentes (Fushimi, et al.,2012). Diferentes fontes de luz foram propostas como alternativas para sistemas a laser, a exemplo dos diodos emissores de luz (LED) que, embora não seja uma fonte monocromática, apresenta uma faixa de emissão mais estreita em comparação com as lâmpadas convencionais (Corazza, et al., 2007).

A fototerapia com Laser de Baixa Intensidade (LBI) é indicada em muitas aplicações biomédicas, inclusive na regeneração tecidual, mostrando vantagens como: o controle da dor, a estimulação do processo de cicatrização, a ação antiinflamatória, o aumento da síntese de colágeno, elastina e fibronectina, proliferação e diferenciação fibroblástica e o aumento da microvascularização local (Melo et al.,2011; Pinheiro et al., 2009; Arany et al., 2007; Pereira et al., 2010; Gonçalves et al., 2013), sendo capaz de induzir a neoangiogênese e a vasodilatação, estimulando a circulação sanguínea em várias patologias e minimizando a destruição tecidual causada pela isquemia e o edema intersticial (Melo et al.,2011; Pereira et al., 2010).

Os efeitos do LBI podem ser observados no comportamento dos linfócitos aumentando sua proliferação e ativação; sobre os macrófagos, aumentando a fagocitose; elevando a secreção de fatores de crescimento de fibroblasto e intensificando a reabsorção tanto de fibrina quanto de colágeno. Além disso, contribuem para elevar a motilidade de células epiteliais, a quantidade de tecido de granulação e, podem diminuir a síntese de mediadores inflamatórios (Andrade et al., 2014).

A efetividade da atividade biológica desse tratamento nos processos fisiológicos e fisiopatológicos, como o reparo tecidual, apresenta benefícios nas três fases do processo cicatricial pelo incremento da adenosina trifosfato (ATP), aceleração do metabolismo celular, aumento na síntese protéica, estimulação da microcirculação local e do sistema linfático que, associada ao aumento da atividade mitótica, resulta na multiplicação celular e formação de novos tecidos e vasos. Dessa forma, contribui para a deposição de fibras colágenas, seja na fase inicial ou tardia, assim como reduz o número de células inflamatórias na fase inicial do processo cicatricial (Melo et al., 2011; Dall et al., 2009).

Afirma-se ainda, que a laserterapia de baixa potência pode gerar aumento da atividade mitocondrial, com consequente aumento de adenosinatrifosfato (ATP), vasodilatação, síntese proteica, decréscimo nos níveis de prostaglandinas, presença de mitose celular, migração e proliferação de queratinócitos e ocorrência do fenômeno de neoangiogênese (Andrade et al., 2014).

Os efeitos do LBI na aceleração da cicatrização de feridas têm sido atribuídos ao estímulo de vários sistemas biológicos, como aumento da proliferação e atividade celular, aumento da síntese de DNA, modulação da produção dos fatores de crescimento e redução na produção de prostaglandinas (Damante, Marques, \& Micheli, 2010).

$\mathrm{Na}$ segunda fase, a neovascularização é reforçada pela fotoestimulação de células endoteliais, com proliferação de fibroblastos e depósito de colágeno, contribuindo para a formação de tecido granulado e o fechamento da ferida (Corazza et al.,2007).

A ação antiinflamatória do LBI é útil para melhorar a cicatrização de feridas por inibir a produção de prostaglandina e a expressão da cicloxigenase, por diminuir os níveis intracelulares de espécies reativas de oxigênio (EROs) e conseqüente aliviar o estresse oxidativo e a morte celular; por promover a eliminação de oxigênio e, dessa forma, proteger contra a morte neuronal bloqueando a via apoptótica mitocondrial induzida pela síntese de óxido nítrico (Lim et al., 2011) e por inibir os fatores quimiotáticos nos estágios iniciais da inflamação (Fiório et al., 2011).

O LBI é capaz de modular eventos oxidativos em tecido lesionado, por dois mecanismos principais: o primeiro resulta na redução na migração e/ou na atividade de células inflamatórias e consequentemente da produção de EROs e o segundo a estimulação da síntese e/ou atividade de enzimas antioxidantes que estão associadas ao controle da expressão gênica de colágeno I e II. No processo de cicatrização normal, embora a produção de EROs aumente, o LBI também aumenta a atividade enzimática antioxidante, minimizando a ocorrência de dano oxidativo. Portanto, acredita-se que o equilíbrio entre a produção 
de EROs e o efeito do sistema antioxidante esteja diretamente relacionado ao tempo de cicatrização e à qualidade do tecido da ferida (Gonçalves et al., 2013; Alster \& Wanitphakdeedecha, 2009).

\section{Resultados e Discussão}

A laserterapia surgiu como uma alternativa para o reparo tecidual, em virtude dos efeitos fotobiomoduladores nos mais diversos mecanismos celulares. No entanto, a literatura é muito controversa: estudos em animais e humanos ora mostram resultados positivos, ora não mostram efeito algum. Essas disparidades podem ser resultantes dos diferentes protocolos e dos parâmetros utilizados para a determinação de seus efeitos. Portanto, mediante a busca sistematizada dos artigos relacionados à temática em questão, selecionamos 10 artigos que resultaram em uma ampla discussão focada nas suas particularidades, abordando a laserterapia como coadjuvante terapeutica no processo de reparo, mediando os fatores que regulam a angiogênese (Tabela 1).

Tabela 1. Análise dos artigos selecionados.

\begin{tabular}{|c|c|c|c|}
\hline Autor, ano & Objetivo & Detalhes do estudo & Conclusão \\
\hline $\begin{array}{l}\text { Carvalho et } \\
\text { al., } 2010\end{array}$ & $\begin{array}{l}\text { Determinar a influência do } \\
\text { LBI (InGaAlP 660nm) na } \\
\text { porcentagem de colágeno e } \\
\text { macrófagos em feridas na } \\
\text { pele em ratos diabéticos }\end{array}$ & $\begin{array}{l}30 \text { ratos wistar machos } \\
\text { Lesão }(8 \mathrm{~mm}) \text { no dorso } \\
\text { GI: diabético tratado com laser } \\
\text { GII: diabético não tratados } \\
\text { Laser: } \mathrm{InGaAlP}(660 \mathrm{~nm}, \mathrm{P}=100 \mathrm{~mW} \text {, } \\
\left.\text { dose }=4 \mathrm{~J} / \mathrm{cm}^{2} \text {, durante } 24 \mathrm{~s} ; \mathrm{DE}=10 \mathrm{~J} / \mathrm{cm}^{2}\right)\end{array}$ & $\begin{array}{c}\text { O laser de baixa potência }(660 \mathrm{~nm}) \\
\text { mostrou-se capaz de influenciar a } \\
\text { porcentagem de colágeno em } \\
\text { feridas na pele aumentando a } \\
\text { quantidade média de fibras de } \\
\text { colágeno e macrófagos }\end{array}$ \\
\hline $\begin{array}{c}\text { Colombo et } \\
\text { al., } 2013\end{array}$ & $\begin{array}{c}\text { Avaliar a angiogênese em } \\
\text { feridas cutâneas dorsais em } \\
\text { um modelo de roedor } \\
\text { tratado com luz laser de } \\
\text { 1660nm. }\end{array}$ & $\begin{array}{c}24 \text { ratos wistar machos } \\
\text { Ferida cutânea excisional } \\
(1 \times 1 \mathrm{~cm}) \text { no dorso } \\
\text { GI: controle } \\
\text { GII: tratado com laser } \\
\text { Laser: } 1660 \mathrm{~nm}, 16 \mathrm{~mW}, 2,5 \mathrm{~J} / \mathrm{cm}^{2} \text { e } 62 \mathrm{~s} / \text { ponto. } \\
\text { Foram } 4 \text { pontos, totalizou } 10 \mathrm{~J} / \mathrm{cm}^{2} \mathrm{e} \\
248 \mathrm{~s} / \text { sessão }\end{array}$ & $\begin{array}{l}\text { O tratamento a laser (1660nm) } \\
\text { contribuiu para aumentar a } \\
\text { angiogênese e influenciar } \\
\text { positivamente a expressão de TGF- } \\
\beta \text { e a deposição de colágeno }\end{array}$ \\
\hline $\begin{array}{l}\text { Fantinati et } \\
\text { al., } 2016\end{array}$ & $\begin{array}{c}\text { Avaliar os efeitos do LBI } \\
\text { na cicatrização de feridas } \\
\text { por queimadura de terceiro } \\
\text { grau em ratos diabéticos e } \\
\text { não diabéticos. }\end{array}$ & $\begin{array}{c}100 \text { ratos wistar fêmeas } \\
\text { Queimaduras com curativos oclusivos } \\
\text { GC: grupo controle } \\
\text { GD: grupo diabético } \\
\text { GTND: grupo tratado não-diabéticos } \\
\text { GTD: grupo tratado diabético } \\
\text { Laser: GaAIAs (650nm/12mW, fluência de } \\
3 \mathrm{~J} / \mathrm{cm}^{2} \text { até o } 7^{\text {a }} \text { dia e } 6 \mathrm{~J} / \mathrm{cm}^{2} \text { até a eutanásia, } \\
\text { dias alternados). }\end{array}$ & $\begin{array}{l}\text { O LBI favoreceu o processo de } \\
\text { cicatrização com } 3 \mathrm{~J} / \mathrm{cm}^{2} \text { na fase } \\
\text { inflamatória e com } 6 \mathrm{~J} / \mathrm{cm}^{2} \text { nas fases } \\
\text { proliferativa e de remodelação, com } \\
\text { efeitos promissores sobre os } \\
\text { diabéticos. }\end{array}$ \\
\hline $\begin{array}{l}\text { Gonçalves et } \\
\text { al., } 2010\end{array}$ & $\begin{array}{l}\text { Determinar os efeitos do } \\
\text { LBI } \\
\text { GaAlAs }\left(30 \text { e } 60 \mathrm{~J} / \mathrm{cm}^{2}\right) \\
\text { GaAs }\left(4 \mathrm{~J} / \mathrm{cm}^{2}\right) \text { em feridas } \\
\text { de ratos }\end{array}$ & $\begin{array}{c}30 \text { ratos wistar machos } \\
\text { Feridas circular }(12 \mathrm{~mm}) \text { dorsolateral } \\
\text { G1(L4): GaAs }\left(904 \mathrm{~nm}, \mathrm{DE}=4 \mathrm{~J} / \mathrm{cm}^{2}\right) \\
\text { G2(L30): GaAlAs }\left(830 \mathrm{~nm}, \mathrm{DE}=30 \mathrm{~J} / \mathrm{cm}^{2}\right) \\
\text { G3(L60): GaAlAs }\left(830 \mathrm{~nm}, \mathrm{DE}=60 \mathrm{~J} / \mathrm{cm}^{2}\right) \\
\text { G4 (D): } 0,1 \mathrm{~g} \text { de pomada, } 1 \mathrm{x} / \mathrm{dia} \\
\text { G5 (controle): } \mathrm{NaCl} 0,9 \%, 1 \mathrm{x} / \mathrm{dia}\end{array}$ & $\begin{array}{c}\text { O LBI com } 30 \mathrm{~J} / \mathrm{cm}^{2} \text { estimulou a } \\
\text { produção de colágeno I e III, } \\
\text { enquanto que } 4 \mathrm{~J} / \mathrm{cm} 2 \text { estimulou a } \\
\text { síntese de colágeno I e velocidade } \\
\text { do fechamento da lesão }\end{array}$ \\
\hline $\begin{array}{l}\text { Martins et } \\
\text { al., } 2015\end{array}$ & $\begin{array}{l}\text { Avaliar a cicatrização de } \\
\text { feridas em ratos usando } \\
\text { LBI associada ao curativo } \\
\text { oclusivo de hidrocolóide }\end{array}$ & $\begin{array}{l}40 \text { ratos wistar machos } \\
\text { Ferida }(0,7 \mathrm{~mm}) \text { no dorso } \\
\text { GI: laser }\left(50 \mathrm{~J} / \mathrm{cm}^{2}(2 \mathrm{~J})-1 \text { dose }\right) \\
\text { GII }(\mathrm{HG}): \text { hidrocolóide } \\
\text { GIII: laser }\left(50 \mathrm{~J} / \mathrm{cm}^{2}(2 \mathrm{~J})-1 \text { dose }+\right. \\
\text { hidrocolóide }) \\
\text { GIV }(\mathrm{CG}): 1 \mathrm{~mL} \text { de } \mathrm{NaCl} 0,9 \% \\
\text { Laser: } 660 \mathrm{~nm}, \mathrm{P}=40 \mathrm{~mW} \text {. }\end{array}$ & $\begin{array}{l}\text { A terapia com LBI, bem como o } \\
\text { curativo hidrocolóide, favoreceram } \\
\text { o processo de cicatrização de } \\
\text { feridas em ratos. }\end{array}$ \\
\hline
\end{tabular}




\begin{tabular}{|c|c|c|c|}
\hline $\begin{array}{c}\text { Matos et al., } \\
2018\end{array}$ & $\begin{array}{l}\text { Avaliar histologicamente o } \\
\text { efeito da fototerapia a laser } \\
\text { (LPT) } \lambda 808 \text { e } \lambda 660 \mathrm{~nm} \text { na } \\
\text { angiogênese no tecido } \\
\text { periodontal de dentes } \\
\text { reimplantados em ratos. }\end{array}$ & $\begin{array}{c}\text { Vinte incisivos superiores direitos foram } \\
\text { extraídos de vinte ratos Wistar e distribuídos } \\
\text { aleatoriamente em quatro grupos }(\mathrm{n}=5) \text { : PN } \\
\text { - os dentes foram armazenados em } \\
\text { guardanapo de papel por } 45 \mathrm{~min} \text {; WM - os } \\
\text { dentes foram imersos em } 20 \mathrm{ml} \text { de leite de } \\
\text { vaca integral UHT por } 45 \mathrm{~min} \text {; PNL e WML - } \\
\text { os dentes receberam o mesmo tratamento } \\
\text { descrito para PN e WM, respectivamente, } \\
\text { mais LPT em } \lambda 808 \text { e } \lambda 660 \mathrm{~nm} \text {. Todos os } \\
\text { canais radiculares foram preparados e } \\
\text { preenchidos com pasta de hidróxido de } \\
\text { cálcio. }\end{array}$ & $\begin{array}{l}\text { LPT em } \lambda 808 \text { e } \lambda 660 \mathrm{~nm} \text { causou } \\
\text { aumento significativo da } \\
\text { angiogênese nos grupos irradiados } \\
\text { (PNL e WML) quando comparado } \\
\text { aos grupos não irradiados (PN e } \\
\text { WM). Portanto a LPT é capaz de } \\
\text { estimular a angiogênese in vivo no } \\
\text { tecido periodontal de dentes } \\
\text { reimplantados. }\end{array}$ \\
\hline $\begin{array}{c}\text { Melo et al., } \\
2011\end{array}$ & $\begin{array}{l}\text { Avaliar o efeito do LBI } \\
\text { (904nm) na cicatrização de } \\
\text { feridas cirúrgicas em ratos. }\end{array}$ & $\begin{array}{c}40 \text { ratos Wistar machos adultos } \\
\text { Incisão na linha Alba com sutura } \\
\text { G1 e G3: não-tratado (eutanásia } 8^{\circ} \text { e } 15^{\circ} \text { dias) } \\
\text { G2 e G4: fotorradiado (eutanásia } 8^{\circ} \text { e } 15^{\circ} \text { dias) } \\
\text { Laser: GaAlAs - } 904 \mathrm{~nm} \text {, aplicação diária, por } \\
2 \text { min, } \mathrm{P}=45 \mathrm{~mW} \text {; Área }=0,02 \mathrm{~cm}^{2}, \mathrm{DE}=3 \mathrm{~J} / \mathrm{cm}^{2} \text {. }\end{array}$ & $\begin{array}{l}\text { A terapia com LBI modula a } \\
\text { resposta inflamatória, com melhor } \\
\text { deposição de fibras colágenas e } \\
\text { aumento do número médio de vasos } \\
\text { neoformados. }\end{array}$ \\
\hline $\begin{array}{c}\text { Feitosa et al., } \\
2015\end{array}$ & $\begin{array}{c}\text { Avaliar os efeitos do LBI } \\
\text { no reparo tecidual em } \\
\text { portadores de úlcera } \\
\text { diabética. }\end{array}$ & $\begin{array}{c}16 \text { pacientes diabéticos de tipo II } \\
\text { Úlcera nos membros inferiores } \\
\text { G1 (controle): assepsia } \\
\text { G2 (experimental): assepsia }+ \text { laser } \\
\text { Laser: } 632,8 \mathrm{~nm}, \mathrm{P}=30 \mathrm{~mW}, \mathrm{DE}=4 \mathrm{~J} / \mathrm{cm}^{2} \text {, por } \\
\text { 80segundos, com } 3 \text { sessoes/semana, em dias } \\
\text { alternados, durante } 1 \text { mês }\end{array}$ & $\begin{array}{l}\text { O LBI parece ser um método } \\
\text { eficiente, viável, indolor e de baixos } \\
\text { custos para o reparo tecidual das } \\
\text { úlceras em pé diabético, com efeitos } \\
\text { biomodulador e analgésico }\end{array}$ \\
\hline $\begin{array}{c}\text { Souza et al., } \\
2015\end{array}$ & $\begin{array}{l}\text { Avaliar o processo de } \\
\text { cicatrização de feridas } \\
\text { cutâneas em camundongos, } \\
\text { utilizando a terapia com } \\
\text { laser AlGaInP em } \\
\text { comparação com o } \\
\text { tratamento com o gerador } \\
\text { de alta frequência }\end{array}$ & $\begin{array}{c}40 \text { camundongos machos } \\
\text { Lesão no dorso }(8 \mathrm{~mm}) \\
\text { G1: controle } \\
\text { Hz, } 120 \mathrm{~s}) \\
\text { G: Gerador de alta frequiência }-\mathrm{HF}(50 / 60 \\
\text { G3: laser AlGaInP }(660 \mathrm{~nm}, \mathrm{P}=30 \mathrm{~mW}, \\
\left.5 \mathrm{~J} / \mathrm{cm}^{2}, 120 \mathrm{~s}\right) \\
\text { G4: laser AlGaInP }(660 \mathrm{~nm}, \mathrm{P}=30 \mathrm{~mW}, \\
\left.8 \mathrm{~J} / \mathrm{cm}^{2}, 120 \mathrm{~s}\right) \\
\text { A primeira irradiação ocorreu } 24 \text { horas após a } \\
\text { lesão, com aplicação diária, } 1 \mathrm{x} / \text { dia, sem } \\
\text { intervalos. }\end{array}$ & $\begin{array}{c}\text { O laser AlGaInP, } 5 \mathrm{~J} / \mathrm{cm}^{2} \text { e } 8 \mathrm{~J} / \mathrm{cm} \text {, } \\
\text { apresentaram melhores resultados } \\
\text { biomodulatórios nas fases aguda e } \\
\text { de remodelação, respectivamente. } \mathrm{O} \\
\mathrm{HF} \text { foi menos eficaz do que o laser, } \\
\text { proporcionando benefícios } \\
\text { significativos somente na fase } \\
\text { inicial do reparo. }\end{array}$ \\
\hline $\begin{array}{c}\text { Tacon et al., } \\
2011\end{array}$ & $\begin{array}{c}\text { Avaliar a atividade } \\
\text { cicatrizante do laser } \\
\text { AlGaInP - 660nm, com } \\
\text { diferentes densidades de } \\
\text { energia, em feridas } \\
\text { cutâneas induzida em ratos. }\end{array}$ & $\begin{array}{c}54 \text { ratos fêmeas wistar } \\
\text { Ferida }(1 \mathrm{~cm}) \text { na região dorso-cervical } \\
\text { G1: controle não-irradiado } \\
\text { G2 (L3): laser e DE }=3 \mathrm{~J} / \mathrm{cm}^{2} \\
\text { G3 }(\mathrm{L} 6) \text { : laser e } \mathrm{DE}=6 \mathrm{~J} / \mathrm{cm}^{2} \\
\text { Laser: AlGaInP }(660 \mathrm{~nm} \text {, modo contínuo, } \\
\text { P=30mW, por 1min, varrendo o leito da } \\
\text { ferida, em dias alternados, iniciando } 25 \mathrm{hrs} \\
\text { após o procedimento cirúrgico). }\end{array}$ & $\begin{array}{c}\text { O laser AlGaInP }\left(3 \mathrm{~J} / \mathrm{cm}^{2} \text { e } 6 \mathrm{~J} / \mathrm{cm}^{2}\right) \\
\text { atuou de forma positiva na } \\
\text { cicatrização devido o aumento da } \\
\text { neovascularização e estímulo à } \\
\text { fibroplasia, assim como pela } \\
\text { redução da resposta inflamatória e } \\
\text { diminuição de PMN e da } \\
\text { hemorragia }\end{array}$ \\
\hline
\end{tabular}

Fonte: Autores.

O processo de cicatrização está sob efeito de várias condições fisiológicas e sistêmicas. No estudo de Carvalho et al., (2010), em se tratando de ratos diabéticos, tem-se a presença de um fator sistêmico que modifica o processo de cicatrização, através de vários fatores como: supressão de reações inflamatórias, angiogênese diminuída, alterações na proliferação e aumento da apoptose dos queratinócitos, diminuição da migração de fibroblastos e defeitos na deposição de colágeno, diminuição da proliferação de células endoteliais e na produção de fatores de crescimento.

A desnutrição tem sido outro aspecto a ser analisado no reparo cicatricial. Dessa forma, os resultados do trabalho de Leite et al., (2014) mostraram que a desnutrição prejudica significativamente a cicatrização cutânea. O uso da fototerapia não promoveu cura ou alterou o colágeno de ratos alimentados, mas acelerou a cicatrização de feridas em ratos nutridos, sugerindo a cura avançada e de forma otimizada. Entretanto, o retardo da cicatrização, ocasionado pela desnutrição, pode ser revertida com o fototerapia. Dessa forma, os autores sugerem a combinação da luz vermelha e infravermelha na faixa de 600 a $1000 \mathrm{~nm}$ 
por acelerar o fluxo sanguíneo local e a inflamação, promovendo a proliferação celular, incluindo células epiteliais, endoteliais, queratinócitos, macrófagos, linfócitos e fibroblastos, aumentando a síntese de colágeno e, em geral, acelerando o reparo tecidual. Além do mais, a situação de subnutrição, corresponde a tipo de condição fisiológica que aumento a sensibilidade das células à absorção e utilização da luz.

A fotobiomodulação a laser promove melhoria no reparo cicatricial em feridas com perda de tecido, um tipo particular de fenômeno conhecido como cicatrização de segunda intenção. No estudo de Melo et al., (2011), as lesões foram suturadas, simulando o que se faz após procedimentos cirúrgicos eletivos. Os resultados mostraram uma redução da intensidade e uma mudança no padrão da reação inflamatória promovida pelo laser e, embora pareça estimular a deposição de fibras de colágeno essa atividade, em feridas suturadas, parece não ser expressiva o suficiente para levar a uma superprodução e formação de cicatrizes hipertróficas. Além disso, a melhor orientação dos feixes de colágeno, bem como a presença de proporções iguais de ambas as fibras de colágeno tipo I e III, parece apontar um papel importante na organização arquitetônica do tecido. O laser também pode atuar estimulando a proliferação de novos vasos sanguíneos e esses efeitos pró-vasculares podem refletir na sobre-estimulação da proliferação endotelial por citocinas liberadas pelas células inflamatórias ou resultantes de estimulação direta do laser no ciclo celular em angioblastos. Entretanto, sugere-se uma estreita relação entre a evolução dos eventos cicatriciais e a redução progressiva da neovascularização durante o processo de cicatrização.

Já no estudo de Martins et al., (2015), a cura da ferida sofreu interferência do curativo hidrocolóide o que explica o resultado do maior tamanho da ferida para o grupo que fez uso só do curativo. Os grupos irradiados mantiveram o mesmo tamanho médio, sugerindo o possível efeito do LBI nos elementos de contração da ferida. Além do mais, as feridas que sofreram radiação se tornam menos edematosas, sugerindo efeito anti-inflamatório pelo LBI. Observou-se que os animais que receberam aplicação do laser associada ou não com aos curativos hidrocolóides estavam mais calmos, sugerindo um efeito analgésico da terapia. A indicação de terapia tópica de lesões com curativo oclusivo para manter a umidade provou e estabeleceu ação no contexto científico, pois mantém a umidade e temperatura ideais para a promoção da ação das células mitóticas, analgesia e desbridamento.

Na pesquisa de Fantinati et al., (2016) foram realizados o desbridamento cirúrgico que contribuiu para a redução da necrose que, por sua vez, permitiu que a luz atinja de forma direta e maiores profundamentes na ferida. O curativo oclusivo foi adotado para manter a hidratação da ferida, minimizando a formação da crosta, traumas e contaminação. Porém o aumento da quantificação da angiogênese e dos fibroblastos pode estar relacionado ao fato do LBI estimular o mecanismo oxidativo das mitocôndrias, favorecendo o aumento significativo no depósito de colágeno e os novos vasos darão suporte à nova matriz intersticial, assim como fornece esse novo ambiente com mais oxigênio e nutrientes, favorecendo a migração de células fibroblásticas que contribuem para o processo de cicatrização.

Com relação à análise macroscópica, as técnicas de microscopia tem sido útil na compreensão dos mecanismos celulares, com o uso de técnicas histológicas, histoquímicas, imuno-histoquímicas e histomorfométricas. A pesquisa de Colombo et al., (2013), pela técnica da imuno-histoquímica com o fator Von Willebrand mostrou menor número de vasos nas primeiras $24 \mathrm{hs}$ no grupo experimental (laserterapia), mesmo assim sendo superior ao grupo controle em todos os períodos, enquanto que a vasodilatação foi notável, especialmente nas primeiras $12 \mathrm{hrs}$ pós-tratamento. Esses dois eventos pode ser explicado devido a degradação dos mastócitos que culmina na redução da formação do vaso e, com relação à vasodilatação, pode-se afirmar que o laser contribuiu para o aumento da área dos vasos, possivelmente devido aos efeitos da luz nos receptores celulares. Com relação ao outro anticorpo, houve aumento da expressão de TGF- $\beta$ ao final do estudo no grupo experimental, o que pode ser considerado um sinal da inibição de respostas imunológicas e inflamatórias, assim como representa um papel improtante na formação da matriz extracelular, com o aumento da deposição de colágeno. 
Diversos comprimentos de ondas, doses e parâmetros tem sido testado na fototerapia com o uso de laser. No estudo de Gonçalves et al., (2010), os dois tipos de laser (GaAs e GaAlAs) parecem indicar a eficiência da radiação na substituição de fibras de colágeno tipo III para o tipo I, sendo os melhores resultado com $4 \mathrm{~J} / \mathrm{cm}^{2}$ e $30 \mathrm{~J} / \mathrm{cm}^{2}$, não encontando bons resultados com as altas densidades. Na pesquisa de Tacon et al., (2011), usando o laser de AlGaInP e comparando baixa densidade $\left(3 \mathrm{~J} / \mathrm{cm}^{2}\right.$ e $\left.6 \mathrm{~J} / \mathrm{cm}^{2}\right)$, os autores sugerem que o aumento do depóstio de colágeno é devido ao estímulo do laser na transformação de fibroblastos em miofibroblastos iniciais, indicando o efeito positivo na fibroplasia. Os efeitos na redução da hemorragia e na diminuição de PMN são decorrentes do efeito positivo do laser na fase da inflamação.

Já no experimento de Souza et al., (2015), comparando fototerapias e diferentes densidades de energias, pode-se inferir que as doses mais baixas do LBI são mais efetivas na fase inicial do processo de cicatrização, enquanto que na fase mais avançada, doses mais altas trarão mais estímulos para a regeneração do tecido. Quanto ao gerador de alta frequencia - HF, embora não tenha apresentado a mesma eficácia que o LBI, seu desempenho também foi mais efetivo na fase inicial de cicatrização. A maior redução de fibroblastos/campo no grupo do laser com a maior densidade de energia sugere um maior estímulo para a maturação de fibroblastos proliferados em colágeno, na presença de doses maiores.

No estudo de Melo et al., (2011), o LBI reduziu a intensidade da reação inflamatória, o que sugere a capacidade de minimizar a resposta inflamatória e favorecer a aceleração de eventos biológicos responsáveis pelo processo de cicatrização, uma vez que induz à mudança do padrão de infiltração de leucócitos (infiltração neutrofílica a linfoplasmocítica) no tecido danificado de feridas irradiadas. Esse efeito modulador sobre a resposta inflamatória pode ser o resultado de um papel inibitório na síntese de prostaglandina, um mediador químico que fornece sinais quimiotáticos de neutrófilo polimorfonucleares, como bem como na proliferação e maturação de linfócitos. Essa terapia resultou ainda em uma deposição reforçada de fibras de colágeno e aumento do número médio de vasos recém-formados.

Matos et al., (2018) utilizando uma combinação de infravermelho ( $\lambda 808 \mathrm{~nm})$ e laser vermelho $(\lambda 660 \mathrm{~nm})$, uma associação mais eficaz na redução do processo inflamatório e na aceleração do reparo tecidual, pois apresentam diferentes absorção e penetração, obtiveram como resultados 15 dias após o reimplante dentário um aumentou significativamente da angiogênese no tecido periodontal em grupos irradiados, indicando um efeito positivo desses comprimentos de onda na cicatrização periodontal de dentes reimplantados. Esses achados indicam que o uso de lasers infravermelhos e vermelhos são capazes de estimular a angiogênese in vivo na pele e feridas orais, resultando em um grande aumento do suprimento de oxigênio e nutrientes para feridas.

Possivelmente, a angiogênese também é induzida pela fototerapia a laser por meio de diferentes mecanismos de sinalização celular. Cury et al. (2013) mostraram que os lasers infravermelhos e vermelhos aumentaram a expressão do fator VEGF, fator induzível por hipóxia (HIF-1 $\alpha$ ) e atividade da metaloproteinase de matriz modulada (MMP-2), que são os três principais mediadores envolvidos na angiogênese. O VEGF desempenha um papel fundamental na angiogênese ao estimular a proliferação e migração de células endoteliais e o HIF-1 $\alpha$ regula a resposta celular à hipóxia, ativando genes que são importantes para a adaptação celular em condições de hipóxia. A atividade da MMP-2 é necessária para a degradação da membrana basal vascular e remodelamento da matriz extracelular, a fim de permitir a migração das células endoteliais durante a angiogênese.

\section{Conclusão}

Diante do exposto, pode-se concluir que a laserterapia tem se apresentado como uma alternativa terapêutica no reparo tecidual, atuando como fotobiomodulador, com efeitos analgésicos, anti-inflamatórios, antiedematosos e antimicrobianos, portanto, oferecendo melhorias nos mecanismos de resposta celular e apresentando vantagens nas três etapas do processo 
cicatricial, seja ela, inflamatória, proliferativa ou remodeladora. Diante disso, é necessária a realização de mais estudos clínicos que demonstre o grande potencial angiogênico da laserterapia em cada fase da cicatrização.

\section{Referências}

Alster, T. S., \& Wanitphakdeedecha, R. (2009). Improvement of postfractional laser erythema with light-emitting diode photomodulation. Dermatologic surgery: official publication for American Society for Dermatologic Surgery, 35(5), 813-815. https://doi.org/10.1111/j.1524-4725.2009.01137.x

Andrade, F., Clark, R. M., \& Ferreira, M. L. (2014). Effects of low-level laser therapy on wound healing. Revista do Colegio Brasileiro de Cirurgioes, 41(2), 129-133. https://doi.org/10.1590/s0100-69912014000200010

Arany, P. R., Nayak, R. S., Hallikerimath, S., Limaye, A. M., Kale, A. D., \& Kondaiah, P. (2007). Activation of latent TGF-beta1 by low-power laser in vitro correlates with increased TGF-beta1 levels in laser-enhanced oral wound healing. Wound repair and regeneration : official publication of the Wound Healing Society [and] the European Tissue Repair Society, 15(6), 866-874. https://doi.org/10.1111/j.1524-475X.2007.00306.x

Carvalho, P., Silva, I. S., Reis, F. A., Perreira, D. M., \& Aydos, R. D. (2010). Influence of ingaalp laser (660nm) on the healing of skin wounds in diabetic rats. Acta cirurgica brasileira, 25(1), 71-79. https://doi.org/10.1590/s0102-86502010000100016

Chaves, M. E., Araújo, A. R., Piancastelli, A. C., \& Pinotti, M. (2014). Effects of low-power light therapy on wound healing: LASER x LED. Anais brasileiros de dermatologia, 89(4), 616-623. https://doi.org/10.1590/abd1806-4841.20142519

Chaves, M. E. A., Silva, F. S. D., Soares, V. P. C., Ferreira, R. A. M., Gomes, F. S. L., Andrade, R. M., \& Pinotti, M. (2015). Evaluation of healing of pressure ulcers through thermography: a preliminary study. Res Biomed Eng, 31 (1), 3-9. https://doi.org/10.1590/2446-4740.0571

Colombo, F., Neto, A., Sousa, A. P., Marchionni, A. M., Pinheiro, A. L., \& Reis, S. R. (2013). Effect of low-level laser therapy ( $\lambda 660$ nm) on angiogenesis in wound healing: a immunohistochemical study in a rodent model. Brazilian dental journal, 24(4), 308-312. https://doi.org/10.1590/0103-6440201301867

Corazza, A. V., Jorge, J., Kurachi, C., \& Bagnato, V. S. (2007). Photobiomodulation on the angiogenesis of skin wounds in rats using different light sources. Photomedicine and laser surgery, 25(2), 102-106. https://doi.org/10.1089/pho.2006.2011

Cury, V., Moretti, A. I., Assis, L., Bossini, P., Crusca, J., Neto, C. B., Fangel, R., de Souza, H. P., Hamblin, M. R., \& Parizotto, N. A. (2013). Low level laser therapy increases angiogenesis in a model of ischemic skin flap in rats mediated by VEGF, HIF-1 $\alpha$ and MMP-2. Journal of photochemistry and photobiology. B, Biology, 125, 164-170. https://doi.org/10.1016/j.jphotobiol.2013.06.004

Dall Agnol, M. A., Nicolau, R. A., de Lima, C. J., \& Munin, E. (2009). Comparative analysis of coherent light action (laser) versus non-coherent light (lightemitting diode) for tissue repair in diabetic rats. Lasers in medical science, 24(6), 909-916. https://doi.org/10.1007/s10103-009-0648-5

Damante, C., Marques, M., \& De Micheli, G. (2010) Terapia com laser em baixa intensidade na cicatrização de feridas-revisão de literatura. $R F O$ - $U P F, 13$ (3), $88-93$.

Fantinati, M. S., Mendonça, D. E. O., Fantinati, A. M. M., Barbosa, D. A., Araújo, L. C., Afonso, C. L., Vinaud, M. C., \& Lino Júnior, R. S.(2016). Activity of low level laser therapy on burning wounds in diabetic rats. Rev bras queimaduras, 15 (1): 42-9.

Feitosa, M. C., Carvalho, A. F., Feitosa, V. C., Coelho, I. M., Oliveira, R. A., \& Arisawa, E. Â. (2015). Effects of the Low-Level Laser Therapy (LLLT) in the process of healing diabetic foot ulcers. Acta cirurgica brasileira, 30(12), 852-857. https://doi.org/10.1590/S0102-865020150120000010

Fiório, F. B., Silveira, L., Jr, Munin, E., de Lima, C. J., Fernandes, K. P., Mesquita-Ferrari, R. A., de Carvalho, P., Lopes-Martins, R. A., Aimbire, F., \& de Carvalho, R. A. (2011). Effect of incoherent LED radiation on third-degree burning wounds in rats. Journal of cosmetic and laser therapy : official publication of the European Society for Laser Dermatology, 13(6), 315-322. https://doi.org/10.3109/14764172.2011.630082

Fushimi, T., Inui, S., Nakajima, T., Ogasawara, M., Hosokawa, K., \& Itami, S. (2012). Green light emitting diodes accelerate wound healing: characterization of the effect and its molecular basis in vitro and in vivo. Wound repair and regeneration : official publication of the Wound Healing Society [and] the European Tissue Repair Society, 20(2), 226-235. https://doi.org/10.1111/j.1524-475X.2012.00771.x

Gonçalves, R. V., Mezêncio, J. M., Benevides, G. P., Matta, S. L., Neves, C. A., Sarandy, M. M., \& Vilela, E. F. (2010). Effect of gallium-arsenide laser, gallium-aluminum-arsenide laser and healing ointment on cutaneous wound healing in Wistar rats. Brazilian journal of medical and biological research $=$ Revista brasileira de pesquisas medicas e biologicas, 43(4), 350-355. https://doi.org/10.1590/S0100-879X2010007500022

Gonçalves, R. V., Novaes, R. D., Cupertino, M., Moraes, B., Leite, J. P., Peluzio, M., Pinto, M. V., \& da Matta, S. L. (2013). Time-dependent effects of lowlevel laser therapy on the morphology and oxidative response in the skin wound healing in rats. Lasers in medical science, 28(2), 383-390. https://doi.org/10.1007/s10103-012-1066-7

Huang, P. J., Huang, Y. C., Su, M. F., Yang, T. Y., Huang, J. R., \& Jiang, C. P. (2007). In vitro observations on the influence of copper peptide aids for the LED photoirradiation of fibroblast collagen synthesis. Photomedicine and laser surgery, 25(3), 183-190. https://doi.org/10.1089/pho.2007.2062

Hussein, A. J., Alfars, A. A., Falih, M. A., \& Hassan, A. N. (2011). Effects of a low level laser on the acceleration of wound healing in rabbits. North American journal of medical sciences, 3(4), 193-197. https://doi.org/10.4297/najms.2011.3193

Iryanov Y. M. (2016). Influence of Laser Irradiation Low Intensity on Reparative Osteogenesis and Angiogenesis Under Transosseous Osteosynthesis. Journal of lasers in medical sciences, 7(3), 134-138. https://doi.org/10.15171/jlms.2016.23

Leite, S. N., Andrade, T. A., Masson-Meyers, D., Leite, M. N., Enwemeka, C. S., \& Frade, M. A. (2014). Phototherapy promotes healing of cutaneous wounds in undernourished rats. Anais brasileiros de dermatologia, 89(6), 899-904. https://doi.org/10.1590/abd1806-4841.20143356 
Lim, W. B., Kim, J. S., Ko, Y. J., Kwon, H., Kim, S. W., Min, H. K., Kim, O., Choi, H. R., \& Kim, O. J. (2011). Effects of 635nm light-emitting diode irradiation on angiogenesis in $\mathrm{CoCl}(2)$-exposed HUVECs. Lasers in surgery and medicine, 43(4), 344-352. https://doi.org/10.1002/1sm.21038

Matos, F. S., Godolphim, F. J., Albuquerque-Júnior, R. L., Paranhos, L. R., Rode, S. M., Carvalho, C. A., \& Ribeiro, M. A. (2018). Laser phototherapy induces angiogenesis in the periodontal tissue after delayed tooth replantation in rats. Journal of clinical and experimental dentistry, 10(4), e335-e340. https://doi.org/10.4317/jced.54499

Martins, S. S., Torres, O. J., Santos, O. J., Limeira Júnior, F., Sauaia Filho, E. N., Melo, S. P., Santos, R. H., \& Silva, V. B. (2015). Analysis of the healing process of the wounds occurring in rats using laser therapy in association with hydrocolloid. Acta cirurgica brasileira, 30(10), 681-685. https://doi.org/10.1590/S0102-865020150100000005

Melo, V. A., Anjos, D. C., Albuquerque Júnior, R., Melo, D. B., \& Carvalho, F. U. (2011). Effect of low level laser on sutured wound healing in rats. Acta cirurgica brasileira, 26(2), 129-134. https://doi.org/10.1590/s0102-86502011000200010

Mendonça, R. J., \& Coutinho-Netto, J. (2009). Cellular aspects of wound healing. Anais brasileiros de dermatologia, 84(3), 257-262. https://doi.org/10.1590/s0365-05962009000300007

Moura, R. O., Nunes, L. C. C., Carvalho, M. E. M., \& Miranda, B. R. (2014). Efeitos da luz emitida por diodos (LED) e dos compostos de quitosana na cicatrização de feridas. Revisão Sistemática. Rev ciênc farm básica apl, 35 (4), 513-518.

Pereira, M. C., de Pinho, C. B., Medrado, A. R., Andrade, Z., \& Reis, S. R. (2010). Influence of 670 nm low-level laser therapy on mast cells and vascular response of cutaneous injuries. Journal of photochemistry and photobiology. B, Biology, 98(3), 188-192. https://doi.org/10.1016/j.jphotobiol.2009.12.005

Pereira A. S., Shitsuka D. M., Shitsuka F. J. P. R. (2018). Metodologia da pesquisa científica.

Pinheiro, A. L., Meireles, G. C., Carvalho, C. M., Ramalho, L. M., \& dos Santos, J. N. (2009). Biomodulative effects of visible and IR laser light on the healing of cutaneous wounds of nourished and undernourished Wistar rats. Photomedicine and laser surgery, 27(6), 947-957. https://doi.org/10.1089/pho.2009.2607

Ribeiro, M. S., Núñez, S. C., Sabino, C. P., Yoshimura, T. M., Silva, C. R., Nogueira, G. E. C., Suzuki, H., \& Garcez, A. S. (2015) Exploring light-based technology for wound healing and appliance disinfection. J Braz Chem Soc, 26 (12), 2583-9.https://dx.doi.org/10.5935/0103-5053.20150253

Sawasaki, I., Geraldo-Martins, V. R., Ribeiro, M. S., \& Marques, M. M. (2009). Effect of low-intensity laser therapy on mast cell degranulation in human oral mucosa. Lasers in medical science, 24(1), 113-116. https://doi.org/10.1007/s10103-007-0531-1

Sousa, R. C., Maia Filho, A. L., Nicolau, R. A., Mendes, L. M., Barros, T. L., \& Neves, S. M. (2015). Action of AlGaInP laser and high frequency generator in cutaneous wound healing. A comparative study. Acta cirurgica brasileira, 30(12), 791-798. https://doi.org/10.1590/S0102-865020150120000001

Tacon, K. C., Santos, H. C., Parente, L. M., Cunha, L. C., Lino-Júnior, R., Ribeiro-Rotta, R. F., Tacon, F. S., \& Amaral, W. N. (2011). Healing activity of laser InGaAlP (660nm) in rats. Acta cirurgica brasileira, 26(5), 373-378. https://doi.org/10.1590/s0102-86502011000500008 